International Journal of Modern Physics A

(C) World Scientific Publishing Company

\title{
NEW CONSTRAINTS ON YUKAWA-TYPE INTERACTIONS FROM THE CASIMIR EFFECT
}

\author{
V. M. MOSTEPANENKO \\ Noncommercial Partnership "Scientific Instruments", Tverskaya Street 11, Moscow, 103905, \\ Russia \\ Vladimir.Mostepanenko@itp.uni-leipzig.de \\ V. B. BEZERRA \\ Department of Physics, Federal University of Paraiba, C.P.5008, CEP 58059-970, João Pessoa, \\ Pb-Brazil \\ G. L. KLIMCHITSKAYA \\ North-West Technical University, Millionnaya Street 5, St.Petersburg, 191065, Russia \\ C. ROMERO \\ Department of Physics, Federal University of Paraíba, C.P.5008, CEP 58059-970, João Pessoa, \\ $\mathrm{Pb}$-Brazil \\ Received 16 November 2011 \\ Revised Day Month Year
}

\begin{abstract}
Measurements of the Casimir force are used to obtain stronger constraints on the parameters of hypothetical interactions predicted in different unification schemes beyond the Standard Model. We review new strong constraints on the Yukawa-type interactions derived during the last two years from recent experiments on measuring the lateral Casimir force, Casimir force in configurations with corrugated boundaries and the Casimir-Polder force. Specifically, from measurements of the lateral Casimir force compared with the exact theory the strengthening of constraints up to a factor of 24 millions was achieved. We also discuss further possibilities to strengthen constraints on the Yukawa interactions from the Casimir effect.
\end{abstract}

Keywords: Yukawa-type interation; Casimir force; non-Newtonian gravity.

PACS numbers: 14.80.-j, 04.50.-h, 04.80.Cc, 12.20.Fv

\section{Introduction}

It is common knowledge that Newton's gravitational law is not tested experimentally with sufficient precision at separations below $0.1 \mathrm{~mm}$. The smaller is the separation between the test bodies, the greater is the correction to Newtonian gravity allowed by the experimental data. For instance, at separation of $1 \mu \mathrm{m}$ the existence of a "correction" that is nine orders of magnitude larger than the magnitude of Newton's 
potential is not excluded experimentally. So large addition scarcely can be called a correction. Because of this it is conventional to speak about non-Newtonian gravity! 1

The most offen discussed non-Newtonian gravity is of Yukawa-type. In this case the complete gravitational potential, including both the Newtonian and nonNewtonian contributions takes the form 1

$$
V(r)=-\frac{G m_{1} m_{2}}{r}\left(1+\alpha e^{-r / \lambda}\right),
$$

where $m_{1}$ and $m_{2}$ are the masses of two pointlike bodies separated by a distance $r, G$ is the Newtonian gravitational constant and $\alpha, \lambda$ are the interaction constant and interaction range of the additional force, respectively.

Modern physics provides two main reasons why the non-Newtonian gravity of Yukawa-type should arise. The first of them is the exchange of light elementary particles between separate atoms of two macrobodies. Many particles, such as, scalar axion, ${ }^{[2]}$ graviphoton, $\stackrel{[3}{,}$ dilaton, $\stackrel{4}{,}$ goldstino,$\underline{[5]}$ and moduli,,$[6$ among others, are predicted in different extensions of the Standard Model. The exchange of light particles of mass $m=\hbar /(\lambda c)$ generates the effective Yukawa-type potential shown by the second term on the right-hand side of Eq. (1). At the present time light elementary particles are regarded as possible constituents of dark matter and dark energy.

Another prediction of Yukawa-type additions to Newton's gravitational law comes from extra-dimensional theories with low-energy compactification scale ${ }^{[7-9}$ According to these theories the compactification energy may be as low as the extradimensional Planck energy

$$
E_{\mathrm{Pl}}^{(D)}=\left(\frac{\hbar^{N+1} c^{N+5}}{G_{D}}\right)^{\frac{1}{2+N}} \sim 1 \mathrm{TeV}
$$

Here $D=4+N, N$ is the number of extra dimensions compactified at some small length $R_{*}, G_{D}=G \Omega_{N}$ is the gravitational constant in the extended $D$-dimensional space-time and $\Omega_{N} \sim R_{*}^{N}$. When $N=0$ one obtains from (2) the usual Planck energy $E_{\mathrm{Pl}}=\left(\hbar c^{5} / G\right)^{1 / 2} \sim 10^{19} \mathrm{GeV}$. The characteristic size of the $N$-dimensional manifold is given by 9

$$
R_{*} \sim \frac{\hbar c}{E_{\mathrm{Pl}}^{(D)}}\left[\frac{E_{\mathrm{Pl}}}{E_{\mathrm{Pl}}^{(D)}}\right]^{\frac{2}{N}} \sim 10^{\frac{32-17 N}{N}} \mathrm{~cm} .
$$

The presence of extra dimensions modifies the standard Newton potential. Thus, at separations $r \gg R_{*}$ the resulting gravitational potential takes the form (1). It is pertinent to compare the theoretical prediction (3) with available experimental data. Thus, for $N=1 \mathrm{Eq}$. (3) leads to $R_{*} \sim 10^{15} \mathrm{~cm}$. Such a large extra dimension is excluded by solar system tests of Newtonian gravitational law 1 For $N=2$ and 3 the sizes of extra dimensions are $R_{*} \sim 1 \mathrm{~mm}$ and $\sim 5 \mathrm{~nm}$, respectively. Keeping in mind that $\lambda \sim R_{*}$, these predictions fall within the range where constraints on non-Newtonian gravity are not so stringent.

Constraints on the Yukawa-type corrections to Newton's law were traditionally obtained from the Eötvos- and Cavendish-type experiments. For this purpose the 
Yukawa interaction energy and force between two test bodies separated by a distance $a$ were obtained by integration of the second term in Eq. (11) over their volumes and subsequent negative differentiation with respect to $a$ :

$$
E_{\mathrm{Yu}}(a)=-G \alpha \rho_{1} \rho_{2} \int_{V_{1}} \int_{V_{2}} d \boldsymbol{r}_{1} d \boldsymbol{r}_{2} \frac{e^{-\left|\boldsymbol{r}_{1}-\boldsymbol{r}_{2}\right| / \lambda}}{\left|\boldsymbol{r}_{1}-\boldsymbol{r}_{2}\right|}, \quad F_{\mathrm{Yu}}(a)=-\frac{\partial E_{\mathrm{Yu}}(a)}{\partial a},
$$

where $\rho_{1}, \rho_{2}$ are the matter densities. The obtained constraints are discussed in detail in the literature. $110 \mid 11$ The strength of these constraints decreases with decreasing $\lambda$. Thus, for the range of the smallest $\lambda$ such that $4.7 \mu \mathrm{m}<\lambda<9 \mu \mathrm{m}$ the maximum allowed value of $\alpha$ varies $12\left[13\right.$ between $3.2 \times 10^{7}$ and $10^{5}$. For $\lambda<4.7 \mu \mathrm{m}$ the strongest constraints on the Yukawa-type interactions follow from measurements of the Casimir force ${ }^{11 \mid 14}$ which becomes the dominant background force at short separations between the test bodies. Note that the possibility to constrain the non-Newtonian gravity from measurements of the van der Waals and Casimir forces was proposed long ago for the Yukawa ${ }^{15}$ and power-type ${ }^{16}$ corrections to Newton's law. A review of constraints on the Yukawa-type interactions from the Casimir effect obtained up to 2009 is available 11

In this paper we review new constraints on non-Newtonian gravity obtained from measurements of the Casimir force during the last two years. During this period a major progress was achieved. Specifically, at the shortest separation range, the strength of high confidence constraints was increased up to a factor of $2.4 \times 10^{7}$. We also consider the prospects of further strengthening these constraints using new experiments on the Casimir force. In Sec. 2 we present constraints obtained from measuring the normal Casimir force between test bodies with smooth surfaces. Section 3 is devoted to constraints following from measurements of the normal Casimir force between test bodies with corrugated surfaces. The constraints following from measurements of the lateral Casimir force are considered in Sec. 4. The constraints obtained from measurements of the thermal Casimir-Polder force are discussed in Sec. 5. Section 6 is devoted to possibilities to further strengthening constraints on the Yukawa interaction using new experimental configurations. Our conclusions are contained in Sec. 7.

\section{Constraints from the Normal Casimir Force Between Test Bodies with Smooth Surfaces}

Here we remind the methodology on how constraints on the Yukawa interactions are obtained from measurements of the normal Casimir force (i.e., acting in the normal direction to the surface). We also present the constraints obtained up to 2009 to be compared with stronger and (or) more recent constraints. As the first such constraint we discuss the one obtained 17 from new torsion pendulum experiment 18

The measured quantities are the Casimir force $F_{C}(a, T)$ in a sphere-plate geometry (experiment using an atomic force microscope ${ }^{19}$ ) and its gradient $F_{C}^{\prime}(a, T)=\partial F_{C}(a, T) / \partial a$ (experiment using a micromachined oscillator $\underline{20 \mid 21}$ ). The 
high-confidence constraints determined at a $95 \%$ confidence level are obtained from the inequalities

$$
\left|F_{Y u}(a)\right| \leq \Xi_{F_{C}}(a), \quad\left|F_{Y u}^{\prime}(a)\right| \leq \Xi_{F_{C}^{\prime}}(a) .
$$

Here $F_{\mathrm{Yu}}(a)$ is the Yukawa force between a sphere (or a spherical lens) and a plate calculated using Eq. (4). The quantity $\Xi_{F_{C}}\left(\Xi_{F_{C}^{\prime}}\right)$ is the half-width of the confidence interval for the difference between theoretical and measured Casimir force (force gradient) determined at a $95 \%$ confidence level. The confidence intervals should not be confused with the force residuals, i.e., the differences between the computed and measured forces (although at least $95 \%$ of force residuals belong to the confidence intervals).

It should be emphasized that $\Xi_{F_{C}}$ and $\Xi_{F_{C}^{\prime}}$ have the meaning of absolute errors. Because of this, they can be determined with only two or three significant figures independently of their values, i.e., with the relative error of about $0.5 \%$. From this it follows that the Yukawa force $F_{\mathrm{Yu}}(a)$ needs not be calculated with a higher precision (even if the measurement of the Casimir force was more precise). It was shown $22 \mid 23$ that the Yukawa force in sphere-plate geometry can be calculated both precisely by Eq. (44) and using the simplified formulation of the proximity force approximation

$$
F_{\mathrm{Yu}}^{s p}(a)=2 \pi R E_{\mathrm{Yu}}^{p p}(a),
$$

where $R$ is the sphere radius and $E_{\mathrm{Yu}}^{p p}(a)$ is the Yukawa energy per unit area in the configuration of two parallel plates. With the experimental parameters used $20 \mid 21$ the results of both calculations coincide. 23

It was claimed 24 that one and the same experiment cannot be used simultaneously to exclude or confirm some theory of the Casimir force and to place constraints on the Yukawa force from the measure of agreement between experiment and theory. This opinion would be warranted only if the difference between the excluded and confirmed theories of the Casimir force can be modeled by the Yukawa force. This is, however, not so in all experiments discussed here. Therefore, it became a common practice in the literature to compare the measured data with different theories and, after a selection of the theory in agreement with the data is made, to obtain constraints on the Yukawa interaction.

In Fig. 1 we present constraints on the parameters of Yukawa-type interaction $(\lambda, \alpha)$ following 2125 from measurements of the normal Casimir force between $\mathrm{Au}$ surfaces by means of an $\mathrm{AFM}^{19}$ (line 1), from measuring the gradient of the Casimir force between similar surfaces (or, equivalently, the Casimir pressure between two Au-coated parallel plates) by means of a micromachined oscillator 20121 (line 2), and from the Casimir-less experiment, $26-28$ where the contribution of the Casimir force was made equal to zero (line 3 ). The constraints for larger $\lambda$ were obtained 29 from the torsion-pendulum experiment 30 of 1997 (line 4). The constraints from the torsion-pendulum experiment 31 of 2009 are shown by the line 5 . The dashed line 6 in Fig. 1 shows the constraints following from the gravitational experiment of Cavendish-type ${ }^{1213}$ which leads to the strongest constraints in this interaction 


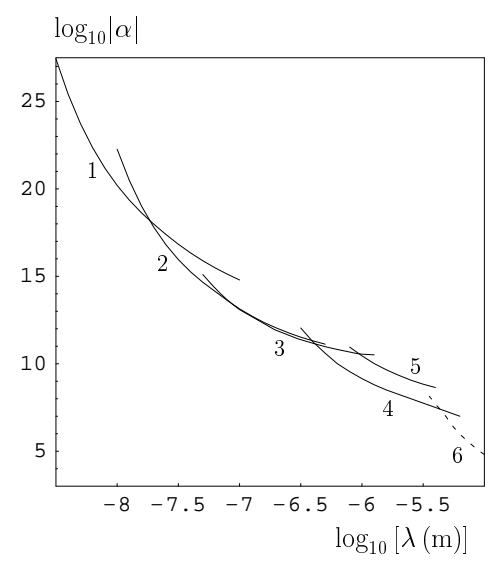

Fig. 1. The constraints on Yukawa-type interactions obtained from measurements of the normal Casimir force between a sphere (spherical lens) and a plate by means of an AFM (line 1), or the Casimir pressure using a micromachined oscillator (line 2), from the Casimir-less experiment (line 3), from the torsion-pendulum experiments of 1997 (line 4) and 2009 (line 5). The dashed line 6 shows the strongest constraints following from gravitational experiments (see text for further discussion).

range. At larger $\lambda$ all the strongest constraints follow from the gravitational experiments as discussed in Sec. 1. In this and in all other figures below, the allowed values of $\lambda$ and $\alpha$ in the $(\lambda, \alpha)$-plane lie beneath the respective lines.

Now let us begin the consideration of new information concerning the constraints shown in Fig. 1 obtained in the last two years. We start with the constraints $\frac{17}{\text { ob- }}$ tained from the measurement data of recent torsion-pendulum experiment, 18 which is similar to previous experiments using the torsion pendulum,, $30 \mid 31$ but arrives at quite different conclusions. This experiment was performed in the geometry of a spherical lens of $R=15.6 \mathrm{~cm}$ radius of curvature near a plate, both coated with Au. The total force of nonestablished origin (presumably caused by large electrostatic patches) between the lens and the plate was up to a factor of 10 larger than the Casimir force even when the residual electric force was compensated. This total force was measured over a wide range of separations from 0.7 to $7.3 \mu \mathrm{m}$. The Casimir force was extracted using the fitting procedure with two fitting parameters. It was found in agreement with the Drude model and in contradiction with the plasma model (contrary to the results of the previous torsion-pendulum experiments $30 \mid 31$ and experiments using a micromachined oscillator $(20 \mid 21)$. The constraints obtained 17 are shown in Fig. 2 by the line 4a. The lines 3, 4, and 6 are the same, as explained in the caption of Fig. 1 and respective discussion in the text. Specifically, line 4 shows the constraints obtained 29 from the first torsion-pendulum experiment 30

The major problem of the recent torsion-pendulum experiment 18 is that at separations above $3 \mu \mathrm{m}$ the Casimir force obtained after the subtraction agrees much better not with the Drude model, as claimed, but with the plasma model ${ }^{32}$ As to separations below $3 \mu \mathrm{m}$, the results of Ref. 18 were shown to be not reliable because 


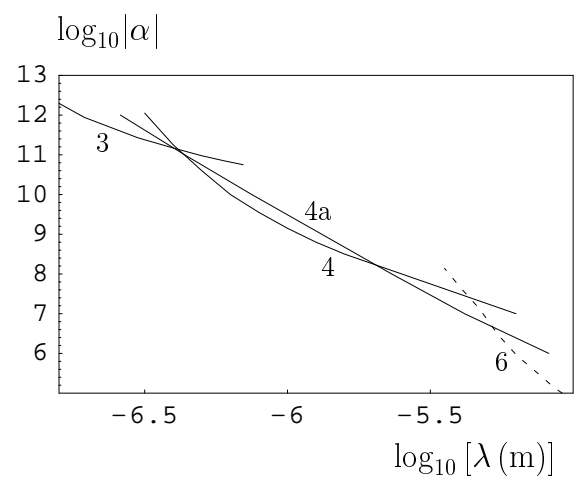

Fig. 2. The constraints on Yukawa-type interactions obtained from the torsion-pendulum experiments of 2011 (line 4a) and 1997 (line 4), and from the Casimir-less experiment (line 3). The dashed line 6 shows the strongest constraints following from gravitational experiments.

of imperfections invariably present on surfaces of large lenses which were not taken into account. 33 As can be seen in Fig. 2, the constraints shown by the lines 4 and 4a are almost of the same strength in spite of the fact that they follow from the two experiments in mutual contradiction. In any case it should be concluded that all constraints following from the torsion-pendulum experiments using large glass lenses (lines 4 and 5 in Fig. 1 and lines 4 and 4a in Fig. 2) are lacking reliability due to the problems indicated in Ref. 33. They are not as reliable as the constraints shown by the lines 1-3 which are deternimed at a $95 \%$ confidence level.

\section{Constraints from the Normal Casimir Force Between Test Bodies with Corrugated Surfaces}

These constraints on the Yukawa-type interaction were obtained ${ }^{34}$ from the results of experiment ${ }^{35}$ measuring the gradient of the normal Casimir force between an Aucoated sphere above a Si plate covered with corrugations of trapezoidal shape (see also Ref. 36). The experimental configuration is schematically shown in Fig. 3(a). The gradient of Yukawa force in this configuration was found in the form 34

$$
F_{\mathrm{Yu}, \mathrm{corr}}^{\prime}(a)=-\left(\frac{p_{1}}{\lambda}+\frac{p_{3}}{H}\right) F_{\mathrm{Yu}}^{s p}\left(a-H_{1}\right)-\left(\frac{p_{2}}{\lambda}-\frac{p_{3}}{H}\right) F_{\mathrm{Yu}}^{s p}\left(a+H_{2}\right) .
$$

Here $p_{i}=l_{i} / \Lambda(i=1,2), p_{3}=1-p_{1}-p_{2}$, and

$$
H_{i}=\frac{\Lambda-l_{i}}{2 \Lambda-l_{1}-l_{2}} .
$$

The Yukawa force between an Au-coated sphere of radius $R$ and a smooth plate is given by 23

$F_{\mathrm{Yu}}^{s p}(z)=-4 \pi^{2} R \alpha G \lambda^{3} e^{-z / \lambda} \rho_{p}\left[\rho_{\mathrm{Au}} \Phi_{1}\left(\frac{\lambda}{R}\right)-\left(\rho_{\mathrm{Au}}-\rho_{s}\right) \Phi_{2}\left(\frac{\lambda}{R}, \frac{\Delta_{\mathrm{Au}}}{R}\right) e^{-\Delta_{\mathrm{Au}} / \lambda}\right]$, 


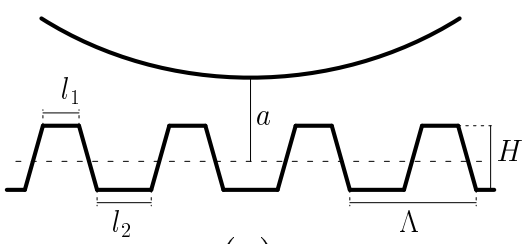

(a)

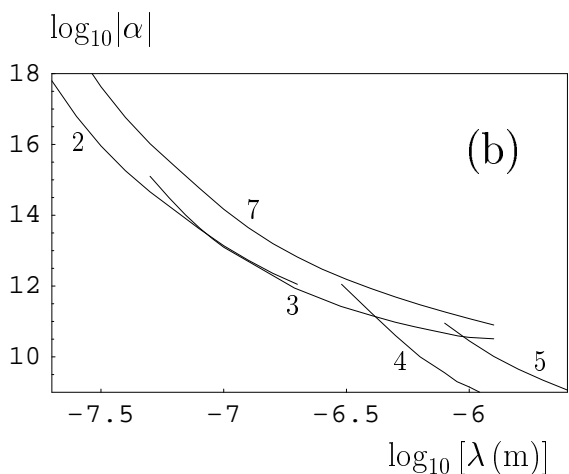

Fig. 3. (a) The configuration of the experiment on measuring the normal Casimir force between a smooth sphere and a plate covered with trapezoidal corrugations. (b) The constraints on Yukawatype interactions obtained from the experiment using a trapezoidally corrugated plate (line 7 ), a micromachined oscillator (line 2), from the Casimir-less experiment (line 3) and from the torsionpendulum experiments of 1997 (line 4) and 2009 (line 5).

where $\rho_{p}, \rho_{s}, \rho_{\mathrm{Au}}$ are the densities of the plate, sphere, and Au, respectively, $\Delta_{\mathrm{Au}}$ is the thickness of the Au coating on the sphere and the functions $\Phi_{1}(x)$ and $\Phi_{2}(x, y)$ are defined as

$$
\begin{aligned}
& \Phi_{1}(x)=1-x+(1+x) e^{-2 / x} \\
& \Phi_{2}(x, y)=1-x-y+(1+x-y) e^{-2(1-y) / x} .
\end{aligned}
$$

Note that under the conditions $x=\lambda / R \ll 1$ and $y=\Delta_{\mathrm{Au}} / R \ll 1$ we have $\Phi_{1,2} \rightarrow 1$ and Eq. (9) takes the form

$$
F_{\mathrm{Yu}, \mathrm{PFA}}^{s p}(a)=2 \pi R E_{\mathrm{Yu}}(a),
$$

where the Yukawa energy per unit area of the two parallel plates is given by the following expression:

$$
E_{\mathrm{Yu}}(a)=-2 \pi \alpha G \lambda^{3} e^{-a / \lambda} \rho_{p}\left[\rho_{\mathrm{Au}}-\left(\rho_{\mathrm{Au}}-\rho_{s}\right) e^{-\Delta_{\mathrm{Au}} / \lambda}\right] .
$$

Equations (11) and (12) were used in calculations of the Yukawa force in the experiments considered in Sec. 2. They lead to the same results for the constraints as obtained from a more exact Eq. (9).

In Fig. 3(b) the constraints on the parameters of Yukawa force following from the experimental data 35 are shown by the line 7 . For comparison purposes, in the same figure the lines 2-5 present the same constraints as in Figs. 1 and 2. As can be seen in Fig. 3(b), the constraints shown by the line 7 are slightly weaker than the constraints shown by lines 2 and 3 . At $\lambda=1.26 \mu \mathrm{m}$, where the minimum difference between the constraints of lines 2 and 3 , on the one hand, and line 7 , on the other hand, is achieved, the constraints of line 7 are weaker by a factor of 2.4. The reason is that the density of $\mathrm{Si}$ is smaller that the density of Au deposited on both test bodies in the respective experiments. $20|21| 26-28$ It is pertinent to note, however, that line 7 in Fig. 3(b) is in a very good qualitative agreement with lines 2 and 3 and, thus, 
provides confirmation to previously obtained constraints. In fact this experiment alone covers the interaction ranges of two previously performed experiments. If the material of the corrugated plate $(\mathrm{Si})$ were replaced by $\mathrm{Au}$, stronger by a factor 8.3 constraints than those shown by line 7 would be obtained (see Sec. 7 where the possibilities to obtain stronger constraints are discussed).

\section{Constraints from the Lateral Casimir Force}

The lateral Casimir force acting between the sinusoidally corrugated surfaces of a sphere and a plate has long been demonstrated and the measurement data were compared with the theory using the PFA. $37 / 38$ Recently more precise measurements of the lateral force were performed and compared with the exact scattering theory 3940 The measure of agreement between the data and theoretical predictions was used 41 to obtain stronger constraints on the Yukawa-type interaction. For this purpose the lateral Yukawa force was calculated in the configuration of a sinusoidally corrugated sphere above a sinusoidally corrugated plate, both coated with $\mathrm{Au}$. The period $\Lambda$ of corrugations on both surfaces was common, but the corrugation amplitudes $A_{1}$ and $A_{2}$ were different. The cause of the appearance of the lateral Casimir force is the phase shift $\varphi$ between corrugations on the sphere and the plate.

The obtained expression for the lateral Casimir force is the following! 41

$$
F_{\text {Yu,lat }}^{\text {sp,corr }}(a, \varphi)=8 \pi^{3} G \alpha \lambda^{3} \Psi(\lambda) e^{-a / \lambda} \frac{A_{1} A_{2}}{b \Lambda} I_{1}\left(\frac{b}{\lambda}\right) \sin \varphi .
$$

Here, $I_{n}(z)$ is the Bessel function of imaginary argument and the quantity $b$ is given by

$$
b \equiv b(\varphi)=\left(A_{1}^{2}+A_{2}^{2}-2 A_{1} A_{2} \cos \varphi\right)^{1 / 2} .
$$

The function $\Psi(\lambda)$ in (13) is defined with account of the layer structure of the sphere and the plate. The corrugated plate of density $\rho_{p}$ was covered with an $\mathrm{Au}$ layer of thickness $\Delta_{\mathrm{Au}, p}$. As to the sphere with density $\rho_{s}$, it was first coated with a Cr layer of density $\rho_{\mathrm{Cr}}$ and thickness $\Delta_{\mathrm{Cr}}$, and then with an Au layer with density

$\rho_{\mathrm{Au}}$ and thickness $\Delta_{\mathrm{Au}, s}$. Taking all this into account, the function $\Psi(\lambda)$ can be presented in the form 41

$$
\begin{aligned}
\Psi(\lambda)= & {\left[\rho_{\mathrm{Au}}-\left(\rho_{\mathrm{Au}}-\rho_{p}\right) e^{-\Delta_{\mathrm{Au}, p} / \lambda}\right]\left[\rho_{\mathrm{Au}} \Phi(R, \lambda)-\left(\rho_{\mathrm{Au}}-\rho_{\mathrm{Cr}}\right) \Phi\left(R-\Delta_{\mathrm{Au}, s}, \lambda\right)\right.} \\
& \left.\times e^{-\Delta_{\mathrm{Au}, s} / \lambda}-\left(\rho_{\mathrm{Cr}}-\rho_{s}\right) \Phi\left(R-\Delta_{\mathrm{Au}, s}-\Delta_{\mathrm{Cr}}, \lambda\right) e^{-\left(\Delta_{\mathrm{Au}, s}+\Delta_{\mathrm{Cr}}\right) / \lambda}\right]
\end{aligned}
$$

where the function $\Phi(x, \lambda)$ is defined as

$$
\Phi(x, \lambda)=x-\lambda+(x+\lambda) e^{-2 x / \lambda} .
$$

The constraints on the parameters of the Yukawa interaction obtained using Eqs. (13)-(16) from the measure of agreement between experiment and theory for the lateral Casimir force are shown in Fig. 4 by line 8. Note that these constraints 


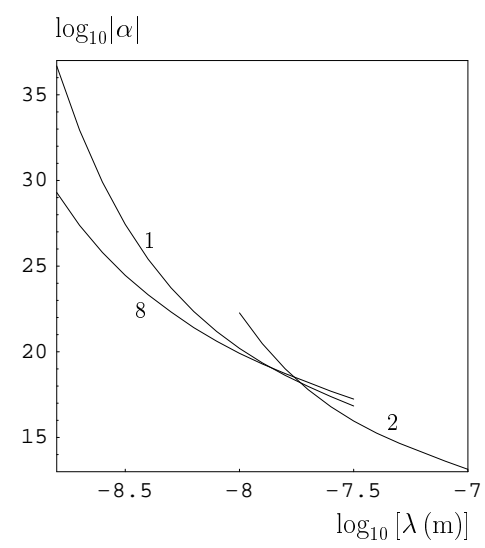

Fig. 4. The constraints on Yukawa-type interactions obtained from measurements of the lateral Casimir force between corrugated surfaces of a sphere and a plate (line 8), from measurements of the normal Casimir force by means of an AFM (line 1) and of the Casimir pressure by means of a micromachined oscillator (line 2).

are determined at the same $95 \%$ confidence level as the total experimental errors in measurements of the lateral Casimir force 3940 The lines 1 and 2 in Fig. 4 are the same as in previous figures. They indicate the strongest high confidence constraints obtained so far. As is seen in Fig. 4, the constraints indicated by line 8 are the strongest over the interaction range from 1.6 to $14 \mathrm{~nm}$. Remarkably, the largest strengthening of previously known constraints shown by line 1 achieves a factor of $2.4 \times 10^{7}$ at $\lambda=1.6 \mathrm{~nm}$. The physical reason for such strong strengthening of the constraints from the experiment with corrugated surfaces is that at a separation, for instance, of $a=121 \mathrm{~nm}$ between the mean levels of corrugations, the distance between the closest points of the surfaces becomes as small as $22 \mathrm{~nm}$. Note that for $\lambda$ below $1 \mathrm{~nm}$ the strongest constraints on the Yukawa interaction are obtained from precision atomic physics. $\frac{42}{4}$

\section{Constraints from the Thermal Casimir-Polder Force}

The thermal Casimir-Polder force between ${ }^{87} \mathrm{Rb}$ atoms and $\mathrm{SiO}_{2}$ plate in the separation region from 6.8 to $11 \mu \mathrm{m}$ was determined 43 from the fractional frequency shift $\gamma_{z}(a)$ of Bose-Einstein condensate oscillations in the direction perpendicular to the plate. The condensate confined in a magnetic trap was characterized by the proper frequency $\omega_{0 z}$ and by the Thomas-Fermi radius $R_{z}$. The experimental data for $\gamma_{z}$ were found $\frac{43}{43}$ to be in agreement with theory disregarding conductivity of $\mathrm{SiO}_{2}$ plate at a constant current (dc conductivity). It was shown 44 also that the data exclude the theory taking into account dc conductivity of $\mathrm{SiO}_{2}$. The constraints on the Yukawa interaction were obtained ${ }^{41}$ from the measure of agreement with theory disregarding dc conductivity of $\mathrm{SiO}_{2}$. For this purpose the fractional frequency shift 


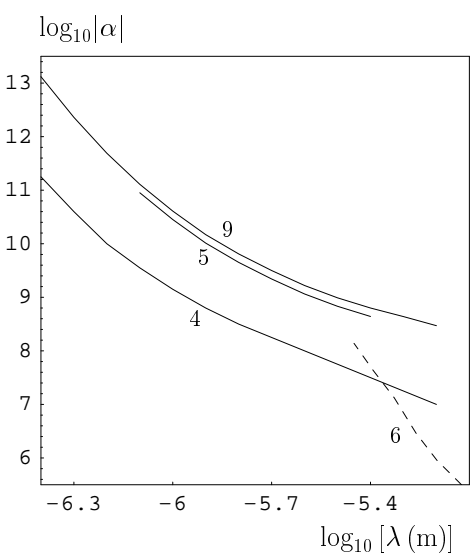

Fig. 5. The constraints on Yukawa-type interactions obtained from measurements of the CasimirPolder force between ${ }^{87} \mathrm{Rb}$ atoms and a plate (line 9), and from the torsion-pendulum experiments of 1997 (line 4) and 2009 (line 5). The dashed line 6 shows the strongest constraints following from gravitational experiments.

due to the Yukawa interaction was found in the form 41

$$
\gamma_{z, \mathrm{Yu}}(a)=\frac{15 \pi G \lambda \rho_{p}}{8 \omega_{0 z}^{2} A_{z}} \alpha e^{-a / \lambda} \Theta\left(\frac{R_{z}}{\lambda}\right) I_{1}\left(\frac{A_{z}}{\lambda}\right)
$$

where

$$
\Theta(t)=\frac{16}{t^{5}}\left(t^{2} \sinh t-3 t \cosh t+3 \sinh t\right) .
$$

The obtained constraints are shown by line 9 in Fig. 5. They are characterized by the same $67 \%$ confidence level, as the experimental error in this experiment. The lines 4 and 5 present the constraints obtained from torsion pendulum experiments. 30131 As discussed in Sec. 2, there are reasons to believe that they are not enough reliable. The line 6 shows the strongest constraints following from gravitational experiments in this interaction range. As can be seen in Fig. 5, the constraints of line 4 are from about 1 to 2 orders of magnitude stronger than the constraints of line 9 . The constraints of line 5 are slightly stronger than the constraints of line 9 obtained from the measurement of Casimir-Polder force. Nevertheless the advantage of the constraints shown by line 9 , as compared with constraints of lines 4 and 5 , is that they are obtained on the basis of an independent measurement and do not use fitting parameters in the comparison between the measured data and theoretical prediction.

\section{Possibilities to Strengthen Constraints on the Yukawa Interactions from Measurements of the Casimir Force}

In the foregoing we have considered new limits on the Yukawa-type interaction obtained during the last two years from experiments on measuring the Casimir force. 
The major strengthening up to a factor of 24 millions was achieved from measurement of the lateral Casimir force between sinusoidally corrugated surfaces of a sphere and a plate. Here we discuss further possibilities to strengthen constraints on the Yukawa interaction using corrugated surfaces. We also discuss the strengthening of constraints on the Yukawa interaction that can be achieved from measuring the gradient of the Casimir force between a microfabricated cylinder and a plate.

\subsection{Constraints from proposed measurements of the gradient of the Casimir force between a smooth sphere and a plate with trapezoidal corrugations}

First we consider the experiment using a smooth sphere and a plate with trapezoidal corrugations considered in Sec. 4. The constraints on Yukawa interaction obtained from this experiment are shown by line 7 in Fig. 3(b). As was discussed in Sec. 4, the reason why these constraints are weaker than the previously obtained (lines 2 and 3) is that the density of $\mathrm{Si}$ is smaller than the density of Au used as the plate material in the experiments of Refs. 20, 21 and 26. Here we preserve the same experimental parameters, as were used in the original experiment, $\frac{35}{b u t}$ replace the Si corrugated plate with a similar plate made of Au. Repeating calculations of the gradient of Yukawa force by Eq. (7) but with $\rho_{p}=\rho_{\text {Au }}$ we obtain 34 the constraints shown by the dashed line in Fig. 6(a). For comparison purposes, in Fig. 6(a) we also plot the same lines 2, 3, 4, 5, and 7 as in Fig. 3(b). As was mentioned in Sec. 4, the constraints from experiment with an Au plate covered with trapezoidal corrugations are stronger by a factor $\rho_{\mathrm{Au}} / \rho_{p} \approx 8.3$ than the constraints of line 7. Within some interaction ranges, the dashed line in Fig. 6(a) also demonstrates stronger constraints than those shown by lines 3,4 , and 5 . Thus, at $\lambda=0.94 \mu \mathrm{m}$, the constraint shown by the dashed line is stronger by a factor of 3.8 than the constraints shown by lines 3 and 5 . At $\lambda=1.04 \mu \mathrm{m}$, the dashed line gives by a factor of 3.5 stronger constraint than that shown by lines 3 and 5 .

\subsection{Constraints from proposed measurements of the normal Casimir force between a smooth sphere and a sinusoidally corrugated plate}

As was discussed in Sec. 2, line 1 in Fig. 1 shows constraints on the Yukawa interaction following from measurement $\$ 19$ of the normal Casimir force between smooth $\mathrm{Au}$ surfaces of a sphere and a plate using an AFM. The constraints of line 1 were significantly improved from measurements of the lateral Casimir force (see line 8 in Fig. 4). The question arises whether the use of corrugated plate in an experiment on measuring the normal Casimir force could lead to stronger constraints. To answer this question, we have calculated the Yukawa force acting between a smooth $\mathrm{Au}$-coated sphere and a sinusoidally corrugated $\mathrm{Au}$ plate 41

$$
F_{\mathrm{Yu}}^{s p, \operatorname{corr}}(a)=-4 \pi^{2} G \alpha \lambda^{3} \Psi(\lambda) e^{-a / \lambda} I_{0}\left(A_{1} / \lambda\right),
$$



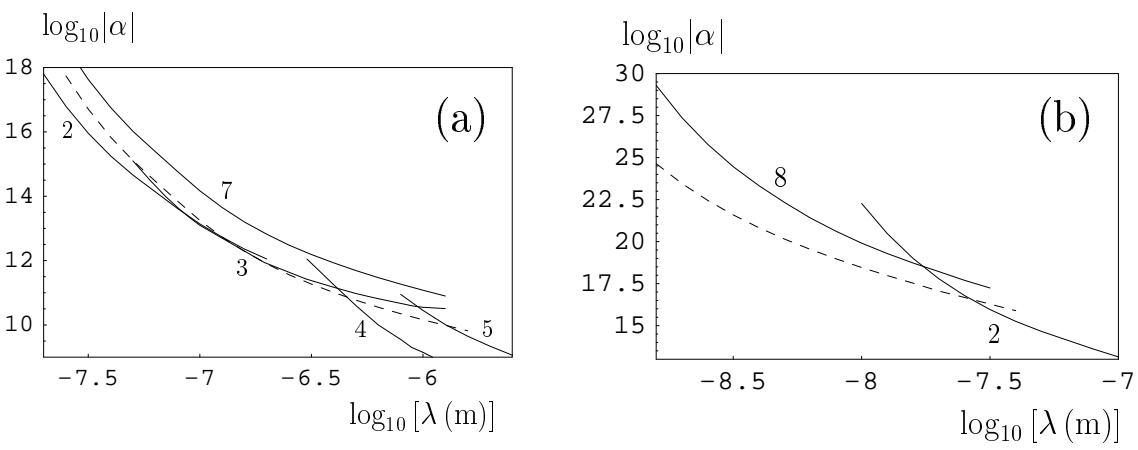

Fig. 6. (a) The prospective constraints on Yukawa-type interactions from measurements of the gradient of the Casimir force between an Au-coated sphere and Au plate covered with trapezoidal corrugations are shown by the dashed line. Lines 2, 3, 4, 5, and 7 are the same as in Fig. 3(b). (b) The prospective constraints from measurements of the normal Casimir force between an Au-coated sphere and Au plate covered with sinusoidal corrugations are shown by the dashed line. Lines 2 and 8 are the same as in Fig. 4.

where $A_{1}$ is the amplitude of corrugations on the plate and the function $\Psi(\lambda)$ is defined in Eq. (15). Note that the sphere is assumed to be coated with only one layer of $\mathrm{Au}$. This means that one should put $\Delta_{\mathrm{Cr}}=0$ in (15). The quantity $\Xi_{F_{C}}(a)$ from Eq. (5) was calculated for this experiment in Ref. 45. In the computations of constraints the experimental parameters of the two experiments $19|39| 40$ were used.

The prospective constraints that can be obtained from this experiment are shown by the dashed line in Fig. 6(b). Lines 8 and 2 show constraints obtained from measurements of the lateral Casimir force using an AFM and the Casimir pressure using a micromechanical oscillator, respectively. As can be seen in Fig. 6(b), the dashed line provides a significant strengthening of the best constraints shown by line 8. Specifically, the largest strengthening by a factor of $4.5 \times 10^{4}$ occurs at $\lambda=1.6 \mathrm{~nm}$. Thus, the use of corrugated surfaces is very prospective for constraining the Yukawa-type interactions.

\subsection{Prospects of using the configuration of a microfabricated cylinder above a plate}

Recently it was proposed to measure the gradient of the Casimir force by means of a micromechanical oscillator in the configurations of a circular $\underline{46}$ or elliptic 47 microfabricated cylinder and a plate. These configurations present some advantages in comparison with configurations of two parallel plates and a sphere above a plate. The Yukawa force acting between a plate and a microfabricated cylinder of length $L$ and radius $R$ can be presented in the form 48

$$
F_{\mathrm{Yu}}^{c p}(a)=-4 \pi^{2} G \rho_{1} \rho_{2} \alpha \lambda^{2} L R e^{-(R+a) / \lambda} I_{1}\left(\frac{R}{\lambda}\right),
$$

where $\rho_{1}$ and $\rho_{2}$ are the densities of the cylinder and plate materials and it is assumed that the thickness of the plate is much larger than $\lambda$. From Eq. (20) for 


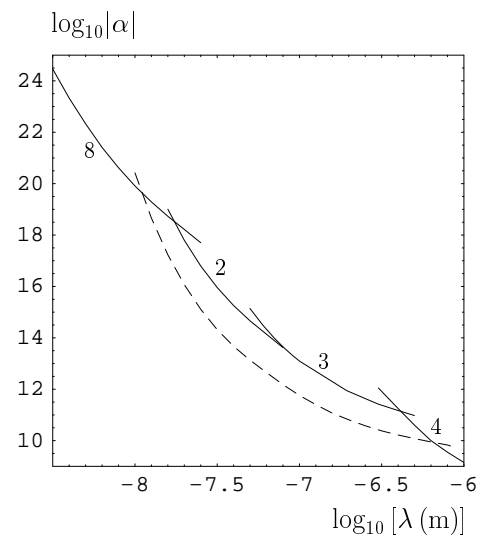

Fig. 7. The prospective constraints on Yukawa-type interactions which can be obtained from measurements of the gradient of normal Casimir force between an Au-coated microfabricated cylinder and an $\mathrm{Au}$-coated plate are shown by the dashed line. Lines 2, 3, 4, and 8 are the same as in Figs. 1 and 4.

the gradient of the Yukawa force one obtains

$$
\frac{\partial F_{\mathrm{Yu}}^{c p}(a)}{\partial a}=4 \pi^{2} G \rho_{1} \rho_{2} \alpha \lambda L R e^{-(R+a) / \lambda} I_{1}\left(\frac{R}{\lambda}\right) .
$$

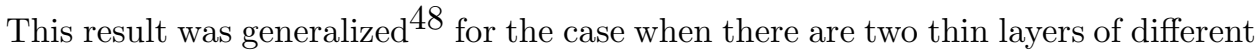
densities covering both the cylinder and the plate.

Equation (21) and its generalizations were used 148 to derive the strongest constraints on Yukawa-type interactions that could be obtained from measurements of the gradient of the Casimir force between a microfabricated cylinder and a plate. The derived constraints are shown by the dashed line in Fig. 7 . The other lines show the strongest constraints obtained from measurements of the lateral Casimir force (line 8), from measurements of the Casimir pressure by means of a micromachined oscillator (line 2), from the Casimir-less experiment (line 3) and from the torsionpendulum experiment of 1997 (line 4). As can be seen in Fig. 7, the proposed experiment promises to strengthen the currently available constraints over a very wide interaction range from $\lambda=12.5 \mathrm{~nm}$ to $\lambda=630 \mathrm{~nm}$. The strongest strengthening, up to 70 times, can be achieved at $\lambda=18 \mathrm{~nm}$. This makes the cylinder-plate geometry promising not only for measurements of the Casimir force, but for obtaining new constraints on non-Newtonian gravity as well.

\section{Conclusions}

As can be concluded from the foregoing, experiments on the Casimir force lead to strongest constraints on Yukawa-type interactions at shorter interaction range where the gravitational experiments fail to provide competitive constraints. One can also conclude that recent measurements of the normal Casimir force between a sphere and a plate with trapezoidal corrugations and of the thermal Casimir- 
Polder force qualitatively confirm constraints obtained from earlier experiments. As to measurements of the lateral Casimir force, it resulted in the strengthening of the previously known high-confidence constraints up to a factor of 24 millions. So strong strengthening has no precedent in other experiments. To conclude, in near future further strengthening of constraints on Yukawa interaction is expected from measuring the Casimir force in configurations with corrugated boundaries and in cylindrical geometries.

\section{Acknowledgments}

V.M.M. and G.L.K. were partially supported by the NSF Grant No. PHY0970161 and by the DFG Grant BO 1112/20-1. V.B.B. and C.R. were partially supported by CNPq.

\section{References}

1. E. Fischbach and C. L. Talmadge, The Search for Non-Newtonian Gravity (Springer, New York, 1999).

2. R. D. Peccei and H. R. Quinn, Phys. Rev. Lett. 38, 1440 (1977).

3. S. Ferrara, J. Scherk and B. Zumino, Nucl. Phys. B 121, 393 (1977).

4. Y. Fujii, Int. J. Mod. Phys. A 6, 3505 (1991).

5. S. Deser and B. Zumino, Phys. Rev. Lett. 38, 1433 (1977).

6. S. Dimopoulos and G. F. Giudice, Phys. Lett. B 379, 105 (1996).

7. I. Antoniadis, N. Arkani-Hamed, S. Dimopoulos and G. Dvali, Phys. Lett. B 436, 257 (1998).

8. N. Arkani-Hamed, S. Dimopoulos and G. Dvali, Phys. Lett. B 429, 263 (1998).

9. N. Arkani-Hamed, S. Dimopoulos and G. Dvali, Phys. Rev. D 59, 086004 (1999).

10. E. G. Adelberger, J. H. Gundlach, B. R. Heckel, S. Hoedl and S. Schlamminger, Progr. Part. Nucl. Phys. 62, 102 (2009).

11. M. Bordag, G. L. Klimchitskaya, U. Mohideen and V. M. Mostepanenko, Advances in the Casimir Effect (Oxford University Press, Oxford, 2009).

12. S. J. Smullin, A. A. Geraci, D. M. Weld, J. Chiaverini, S. Holmes and A. Kapitulnik, Phys. Rev. D 72, 122001 (2005).

13. A. A. Geraci, S. J. Smullin, D. M. Weld, J. Chiaverini and A. Kapitulnik, Phys. Rev. D 78, 022002 (2008).

14. G. L. Klimchitskaya, U. Mohideen and V. M. Mostepanenko, Rev. Mod. Phys. 81, 1827 (2009).

15. V. A. Kuzmin, I. I. Tkachev and M. E. Shaposhnikov, Pis'ma v ZhETF 36, 49 (1982) [JETP Lett. 36, 59 (1982)].

16. V. M. Mostepanenko and I. Yu. Sokolov, Phys. Lett. A 125, 405 (1987).

17. A. O. Sushkov, W. J. Kim, D. A. R. Dalvit and S. K. Lamoreaux, Phys. Rev. Lett. 107, 171101 (2011).

18. A. O. Sushkov, W. J. Kim, D. A. R. Dalvit and S. K. Lamoreaux, Nature Phys. 7, 230 (2011).

19. B. W. Harris, F. Chen and U. Mohideen, Phys. Rev. A 62, 052109 (2000).

20. R. S. Decca, D. López, E. Fischbach, G. L. Klimchitskaya, D. E. Krause and V. M. Mostepanenko, Phys. Rev. D 75, 077101 (2007).

21. R. S. Decca, D. López, E. Fischbach, G. L. Klimchitskaya, D. E. Krause and V. M. Mostepanenko, Eur. Phys. J. C 51, 963 (2007). 
22. R. S. Decca, E. Fischbach, G. L. Klimchitskaya, D. E. Krause, D. López and V. M. Mostepanenko, Phys. Rev. D 79, 124021 (2009).

23. E. Fischbach, G. L. Klimchitskaya, D. E. Krause and V. M. Mostepanenko, Eur. Phys. J. C 68, 223 (2010).

24. A. Lambrecht and S. Reynaud, arXiv:1106.3848v1.

25. E. Fischbach, D. E. Krause, V. M. Mostepanenko, and M. Novello, Phys. Rev. D 64, 075010 (2001).

26. R. S. Decca, D. López, H. B. Chan, E. Fischbach, D. E. Krause and C. R. Jamell, Phys. Rev. Lett. 94, 240401 (2005).

27. R. S. Decca, D. López, H. B. Chan, E. Fischbach, G. L. Klimchitskaya, D. E. Krause and V. M. Mostepanenko, J. Low Temp. Phys. 135, 63 (2004).

28. C. R. Jamell and R. S. Decca. Int. J. Mod. Phys. A 26, 3742 (2011).

29. M. Bordag, B. Geyer, G. L. Klimchitskaya, and V. M. Mostepanenko, Phys. Rev. D 58, 075003 (1998).

30. S. K. Lamoreaux, Phys. Rev. Lett. 78, 5 (1997).

31. M. Masuda and M. Sasaki, Phys. Rev. Lett. 102, 171101 (2009).

32. G. L. Klimchitskaya, M. Bordag, E. Fischbach, D. E. Krause and V. M. Mostepanenko, Int. J. Mod. Phys. A 26, 3918 (2011); Int. J. Mod. Phys.: Conf. Ser. 3, 515 (2011).

33. V. B. Bezerra, G. L. Klimchitskaya, U. Mohideen, V. M. Mostepanenko and C. Romero, Phys. Rev. B 83, 075417 (2011).

34. V. B. Bezerra, G. L. Klimchitskaya, V. M. Mostepanenko and C. Romero, Phys. Rev. D 83, 075004 (2011).

35. Y. Bao, R. Guérout, J. Lussange, A. Lambrecht, R. A. Cirelli, F. Klemens, W. M. Mansfield, C. S. Pai, and H. B. Chan, Phys. Rev. Lett. 105, 250402 (2010).

36. G. L. Klimchitskaya, U. Mohideen and V. M. Mostepanenko, Int. J. Mod. Phys. B 25, 171 (2011).

37. F. Chen, U. Mohideen, G. L. Klimchitskaya and V. M. Mostepanenko, Phys. Rev. Lett. 88, 101801 (2002).

38. F. Chen, U. Mohideen, G. L. Klimchitskaya and V. M. Mostepanenko, Phys. Rev. A 66, 032113 (2002).

39. H.-C. Chiu, G. L. Klimchitskaya, V. N. Marachevsky, V. M. Mostepanenko and U. Mohideen, Phys. Rev. B 80, 121402(R) (2009).

40. H.-C. Chiu, G. L. Klimchitskaya, V. N. Marachevsky, V. M. Mostepanenko and U. Mohideen, Phys. Rev. B 81, 115417 (2010).

41. V. B. Bezerra, G. L. Klimchitskaya, V. M. Mostepanenko and C. Romero, Phys. Rev. D 81, 055003 (2010).

42. S. G. Karshenboim, Phys. Rev. D 82, 073003 (2010).

43. J. M. Obrecht, R. J. Wild, M. Antezza, L. P. Pitaevskii, S. Stringari and E. A. Cornell, Phys. Rev. Lett. 98, 063201 (2007).

44. G. L. Klimchitskaya and V. M. Mostepanenko, J. Phys. A 41, 312002(F) (2008).

45. G. L. Klimchitskaya, U. Mohideen and V. M. Mostepanenko, J. Phys. A 40, F339 (2007).

46. R. S. Decca, E. Fischbach, G. L. Klimchitskaya, D. E. Krause, D. López and V. M. Mostepanenko, Phys. Rev. A 82, 052515 (2010).

47. R. S. Decca, E. Fischbach, G. L. Klimchitskaya, D. E. Krause, D. López and V. M. Mostepanenko, Phys. Rev. A 84, 042502 (2011).

48. G. L. Klimchitskaya and C. Romero, Phys. Rev. D 82, 115005 (2010). 